\title{
Design and Simulation of A Rectifier Controller Based on Fuzzy PID
}

\author{
Yunfeng Ni \\ School of Electrical Engineering, Xi' an University of \\ Science \& Technology Xi' an, 710054 \\ Xi'an ,China \\ e-mail: niyunfeng@xust.edu.cn
}

\author{
Junliang Zhang \\ School of Electrical Engineering, Xi' an University of \\ Science \& Technology, Xi’an, 710054 \\ Xi' an ,China \\ e-mail: zhangjunliang2008@163.com
}

\begin{abstract}
With the disadvantages of large time-varying, non-linearity and uncertainty, it is hard to establish a precise mathematical model for the current $D C$ thyristor rectifier equipment load which results in conventional PID control performance degradation. A control strategy based on fuzzy PID advanced control theory is proposed. According to expert control rules decision table to determine the size of control quantity, this strategy possesses flexibility and robustness of fuzzy control, and high accuracy of traditional PID control. Using the ARM+FPGA architecture of the embedded system to achieve control strategy, controlling precision and speed have good improvement compared with the traditional single processor design scheme. By simulating 12 pulse rectification control circuitry in MATLABSIMULINK, results show that compared to conventional PID, the fuzzy PID control system has smaller overshoot, faster dynamic response and greater stability. It can realize efficient control of controller objects of large power rectifier electronics equipment which have varied application and changing load. In the industrial control and large current power electric field, this method has wide application prospect.
\end{abstract}

Keywords-Fuzzy PID; Rectifier; Trigger pulse; Simulation

\section{INTRODUCTION}

In the power electronic converters field of electroplating, electrolysis, induction heating, metallurgy, etc, rectifier equipment plays an important role. Currently, most of the industrial control field used rectifier equipment is based on thyristor controlled rectifier phase technique. The most critical part generates trigger pulse and control systems. However, since the rectifier equipment DC loads have large variability, nonlinearity and uncertainty, so that the control performance of traditional PID control technology rectifier equipment declined significantly[1]. To solve these problems, in the rectifier control system, it is taken advanced PID control algorithm fuzzy control strategy, without need to establish a precise mathematical model of the controlled object. According to the expert control rules decision table, it can determine the controlled quantity, with fuzzy flexibility and robustness of traditional PID control and high control accuracy. Compared to conventional PID control method, fuzzy PID control system has smaller overshoot, faster dynamic response and greater stability, can realize effective control high-power electronic rectifier equipment such varied applications, changing load.

\section{THE OVERALL STRUCTURE}

Rectifier control device is composed of main circuit and the control circuit which can realize AC / DC conversion. In this paper,12-pulse rectifier power phase shifted main circuit drop the impact of rectifier circuit on the grid, with a high power factor, reduce effectively the power grid harmonic interference. The design of its control system is shown as Fig. 1.Rectifier circuit is divided into the main circuit and control circuits. Main circuit consists of 12 thyristors, every full-controlled bridge consisted of 6-pulse rectifier circuit I, II is made up of six thyristor, all the controlled bridge I, II series connection and constitute the entire main circuit. The control circuit consists of generation the signal conditioning section, a synchronization signal, fuzzy PID control section, 12-pulse trigger control and other components[2][3].

According to the theory of power electronic rectifier it can get DC output voltage[4]:

$$
U_{d}=\frac{6 \sqrt{6} U_{2}}{\pi} \cos \alpha
$$

In (1) , U2 is effective voltage value of the secondary side in the transformer, Ud is output DC voltage of the rectifier side, and $\alpha$ is phase shifted angle.

Control strategy is as follows: it gets reference point from three-phase synchronous phase trigger pulse signal, and gets the feedback signal from the rectifier side current sensor[5]. Due to the Hall device output current is small, typically value is $\mathrm{mA}$ level, therefore, it is necessary for designing the signal conditioning circuitry, signal conditioning through STM32 microprocessor to achieve AD sampling. Fuzzy PID processes the value of AD, and generates the trigger angle $\alpha$. It's programmed with 12 trigger pulse thyristor by the trigger angle and pulse synchronization signal in FPGA[6]. So the control system can achieve rectifier phase shifting digital controlling.

\section{FUZZY PID CONTROL OF RECTIFER}

Fuzzy control is a kind of computer control method that based on fuzzy setting theory, fuzzy linguistic variables, and fuzzy logic. Fuzzy PID control algorithm combined with fuzzy control algorithm and classical PID control algorithm. First of all, through analyzing the system stability, response speed, overshoot, and steadystate identifies precision PID parameters. Secondly derived the impact of $\mathrm{Kp}, \mathrm{Ki}, \mathrm{Kd}$ with system output characteristics, established the relations of three PID parameters $\mathrm{Kp}, \mathrm{Ki}, \mathrm{Kd}$ 
with error e and error changing rate ec. Through continuous testing e, ec and combining with expert experience and fuzzy rules for fuzzy reasoning, study fuzzy rules for $\mathrm{Kp}, \mathrm{Ki}$, Kd with adjusting online. Reaches different e, ec, control the PID parameters. Thus controlled object has more better dynamic performance to classic PID control. Fuzzy PID control has a smaller overshoot and faster, dynamic response and greater stability, has good application prospects.
(2) When $\mid$ e $\mid$ is medium large, in order to respond with a smaller overshoot, $\mathrm{Kp}$ values is smaller, in order to have better response rate, $\mathrm{Ki}, \mathrm{Kd}$ values are moderate.

(3) When | e | is medium, for the system in order to have a good steady-state performance, should take larger $\mathrm{Kp}$, Ki. When | ec | is small, Kd generally medium-sized area. When $\mid$ ec $\mid$ is larger, Kd values are usuallysmall.

C Fuzzy Adaptive PID algorithm design

1) Fuzzy input and output variable

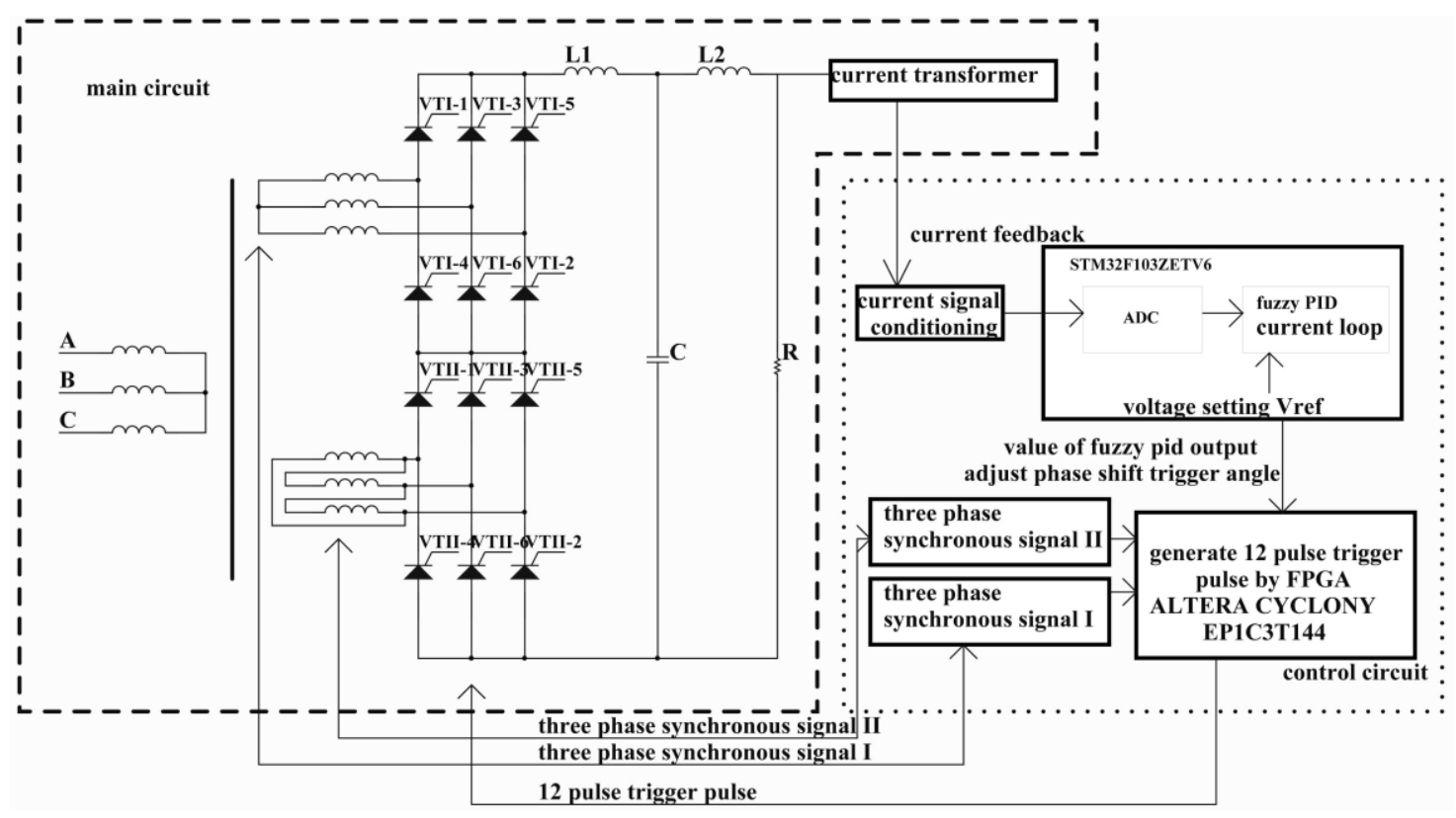

Figure 1. the overall struture

\section{A. Fuzzy PID control}

The structure of fuzzy PID controller is shown in Fig. 2, The system of three two-input-one-output fuzzy controller. The input variable is the error e and ec, ec is the changing rate of e. The output variable is the real time current value $i$ of the controlled object. It can adjust the PID controller $\mathrm{Kp}, \mathrm{Ki}, \mathrm{Kd}$. The controller works as follows: according to the current error e, changing error rate ec, fuzzy inference, vague solutions, we get $\triangle \mathrm{Kp}, \triangle \mathrm{Ki}$, and $\triangle \mathrm{Kd}$ which are the changing quantities of the three parameter to control the controlled object.

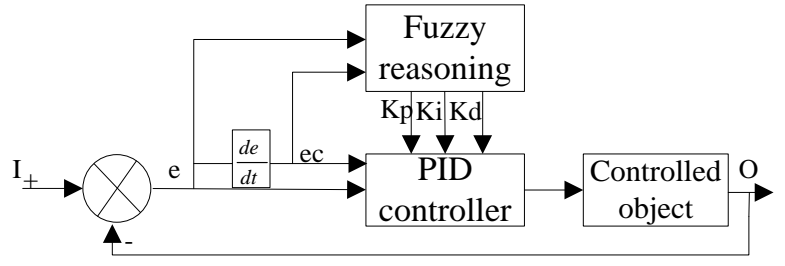

Figure 2. the structure of fuzzy PID controller

\section{B. Fuzzy adaptive PID parameter setting principle [7]}

On the basis of expertise in different | e || ec | when self-tuning PID parameters have the following rules:

(1) when $|\mathrm{e}|$ is large, in order to make the system better fast tracking performance, should take a larger Kp and a smaller $\mathrm{Kd}$, in order to avoid a larger overshoot response, $\mathrm{Ki}$ is less.
Fuzzification is a process that mapping precise variable which is the observation of the input space to fuzzy set in fuzzy domain. Because the fuzzy controller can only deal with the fuzzy quantity, so the exact input must be fuzzy, and convert to fuzzy value, available for fuzzy controlling. In this control system, the input variable is the error e, the error rate of change ec, the output variable is $\triangle \mathrm{Kp}, \triangle \mathrm{Ki}, \triangle \mathrm{Kd}$. The error e and error change rate ec range as fundamental domain, labeled [-e,e] [- ec, ec], range are $[6,6]$. The fuzzy subset of $e=\{N B, N M, N S$, $\mathrm{ZO}, \mathrm{PS}, \mathrm{PM}, \mathrm{PB}\}$, fuzzy error rate of change set for the ec $=\{\mathrm{NB}, \mathrm{NM}, \mathrm{NS}, \mathrm{ZO}, \mathrm{PS}, \mathrm{PM}, \mathrm{PB}\}$.

$\mathrm{NB}$ indicates that the variable is negative and the absolute value is the maximum, the range of value is [-6, $5)$;

NM indicates that the variable is negative and absolute value is medium, the range of value is $[-5,-3)$;

NS indicates that the variable is negative and the absolute value is the smaller, the range of value is $[-3,-1)$;

$\mathrm{O}$ indicates that the variable is zero, and the range of value is $[-1,1)$,

PS indicates that the variable is positive and the absolute value is the smaller, the range of value is $[1,3)$;

$\mathrm{PM}$ indicates that the variable is positive and absolute value is medium, the range of value is $[3,5)$;

PB indicates that the variable is positive and the absolute value is the maximum, the range of value is $[5,6]$;

System error e, error changing rate ec and output variables are selected triangular membership functions.

2) The table of fuzzy PID controller. 
The fuzzy control rules are composed of a series of "IF-THEN" type fuzzy conditional statements. By constantly summing up experience experts, fuzzy control rules are shown as table 1 .

TABLE I. FUZZY PID CONTROL RULES

\begin{tabular}{|c|c|c|c|c|c|c|c|c|}
\hline \multirow{2}{*}{\multicolumn{2}{|c|}{ KpKiKd }} & \multicolumn{7}{|c|}{$\mathrm{e}$} \\
\hline & & NB & NM & NS & $\mathrm{O}$ & PS & PM & PB \\
\hline \multirow{7}{*}{ e } & NB & $\mathrm{NB} / \mathrm{O} / \mathrm{PS}$ & $\mathrm{NB} / \mathrm{PS} / \mathrm{PS}$ & $\mathrm{PM} / \mathrm{PB} / \mathrm{PM}$ & $\mathrm{PS} / \mathrm{PB} / \mathrm{PB}$ & PM/PLPM & $\mathrm{NB} / \mathrm{PS} / \mathrm{PS}$ & $\mathrm{PB} / \mathrm{O} / \mathrm{PS}$ \\
\hline & NM & $\mathrm{NB} / \mathrm{O} / \mathrm{PS}$ & NB/PS/PS & $\mathrm{PM} / \mathrm{PB} / \mathrm{PM}$ & PS/PB/PB & PM/PL/PM & NB/PS/PS & $\mathrm{PB} / \mathrm{O} / \mathrm{PS}$ \\
\hline & NS & $\mathrm{NB} / \mathrm{O} / \mathrm{PS}$ & PM/O/PM & $\mathrm{PS} / \mathrm{PM} / \mathrm{PB}$ & $\mathrm{O} / \mathrm{PB} / \mathrm{PB}$ & $\mathrm{PS} / \mathrm{PM} / \mathrm{PB}$ & $\mathrm{PM} / \mathrm{O} / \mathrm{PM}$ & $\mathrm{PB} / \mathrm{O} / \mathrm{PS}$ \\
\hline & $\mathrm{O}$ & $\mathrm{NB} / \mathrm{O} / \mathrm{PS}$ & PM/O/PM & $\mathrm{PS} / \mathrm{PM} / \mathrm{PB}$ & $\mathrm{O} / \mathrm{PB} / \mathrm{PB}$ & $\mathrm{PS} / \mathrm{PM} / \mathrm{PB}$ & $\mathrm{PM} / \mathrm{O} / \mathrm{PM}$ & $\mathrm{PB} / \mathrm{O} / \mathrm{PS}$ \\
\hline & PS & $\mathrm{NB} / \mathrm{O} / \mathrm{PS}$ & $\mathrm{PM} / \mathrm{O} / \mathrm{PM}$ & $\mathrm{PS} / \mathrm{PM} / \mathrm{PB}$ & $\mathrm{O} / \mathrm{PB} / \mathrm{PB}$ & $\mathrm{PS} / \mathrm{PM} / \mathrm{PB}$ & $\mathrm{PM} / \mathrm{O} / \mathrm{PM}$ & $\mathrm{PB} / \mathrm{O} / \mathrm{PS}$ \\
\hline & PM & NB/O/PS & $\mathrm{PM} / \mathrm{PS} / \mathrm{PS}$ & $\mathrm{PM} / \mathrm{PB} / \mathrm{PM}$ & $\mathrm{PS} / \mathrm{PB} / \mathrm{PB}$ & $\mathrm{PM} / \mathrm{PB} / \mathrm{PM}$ & $\mathrm{PB} / \mathrm{PB} / \mathrm{PS}$ & $\mathrm{PB} / \mathrm{O} / \mathrm{PS}$ \\
\hline & PB & NB/O/PS & $\mathrm{PM} / \mathrm{PS} / \mathrm{PS}$ & $\mathrm{PM} / \mathrm{PB} / \mathrm{PM}$ & $\mathrm{PS} / \mathrm{PB} / \mathrm{PB}$ & $\mathrm{PM} / \mathrm{PB} / \mathrm{PM}$ & $\mathrm{PB} / \mathrm{PS} / \mathrm{PS}$ & $\mathrm{PB} / \mathrm{O} / \mathrm{PS}$ \\
\hline
\end{tabular}

In the MATLAB fuzzy logic tools, we gets fuzzy inference rules curved of three parameters about fuzzy PID controller, as shown as Fig. 3(a) (c).

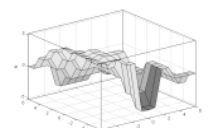

(a) $\triangle \mathrm{Kp}$

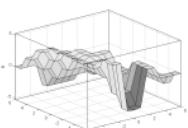

(b) $\triangle \mathrm{Ki}$

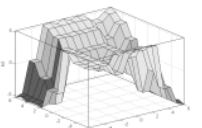

(c) $\triangle \mathrm{Kd}$
Figure 3. fuzzy inference rules curved

3) Fuzzy reasoning and defuzzification.

Fuzzy reasoning is the core element of fuzzy controller. It is based on the concept of fuzzy reasoning ability to simulate the human. By fuzzy inputs and fuzzy control rules, the fuzzy control system completes fuzzy inference, using Mamdani fuzzy reasoning algorithm to obtain the fuzzy amount of fuzzy controller controller. The amount of the resulting fuzzy inference must be converted into a clear solution, it can be used to control the phase-shifted trigger pulse. The design uses a summation of gravity defuzzification method complete defuzzification.

\section{SYSTEM SIMULATION STUDY}

System Modeling and Simulation. The control system controlled objects thyristor rectifier are divided into three parts, thyristor rectifier circuit, filter circuit and DC loads. The dynamic model of DC circuit for thyristor rectifier with balance device can be equivalent to an order reactor inertia[8], as shown in (2).

$$
G_{x}(s)=\frac{U_{d}(s)}{\alpha(s)}=\frac{K}{1+T_{S} s}
$$

In (2) ,Ud is the output DC voltage of the rectifier side, and $\alpha$ is phase shifted angle. The value of Ts is determined by the form of the rectifier circuit. $\mathrm{K}$ is the ratio value with the rated voltage rectifier output and the maximum input voltage by the rectifier firing angle.

The circuit of resistance inductive load shown in Figure 4.According to the circuit theory, we know the transfer function of the load resistance and resistive inductive load transfer functions such as (3) and (4).

$$
G_{R}(s)=\frac{U_{d}(s)}{I_{d}(s)}=\frac{R C s+1}{R L C s^{2}+L s+R}
$$

$G_{z}(s)=\frac{U_{d}(s)}{I_{d}(s)}=\frac{L_{2} C s^{2}+R C s+1}{L_{1} L_{2} C s^{3}+R L_{1} C s^{2}+L_{1} s+L_{s} s+R}$

For fuzzy PID control, it does not need to establish a precise mathematical model of controlled object, so the thyristor rectifier systems usually simulate purely resistive load with resistance, inductance, resistor in series with the analog resistive inductive load.

Controlled object transfer is indicated in (2)[9]: $\mathrm{K}=$ $44 \mathrm{Ts}=0.00167 \mathrm{R}=1 \Omega \mathrm{C}=33 \mathrm{mF} \mathrm{L}=3.5 \mathrm{mH}$. In order to simplify the simulation model, since $\mathrm{Ts} \ll 1$, so we take Ts $=0$, thus the transfer function of controlled objects can be obtained such as (5).

$$
\mathrm{H}(\mathrm{s})=\frac{300 s+10000}{s^{2}+35 s+10000}
$$

It's established the Fuzzy PID control system simulation model of rectifier showing in Fig. 4 (a) (d).

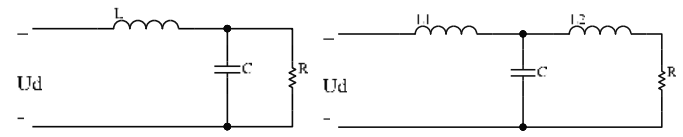

(a) resistive load resistance inductive load

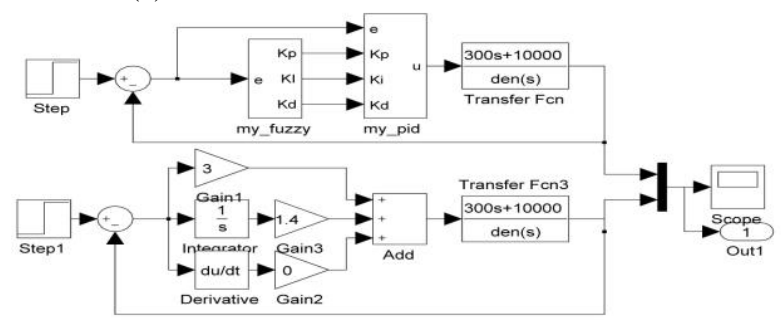

(b) compared fuzzy and conventional PID

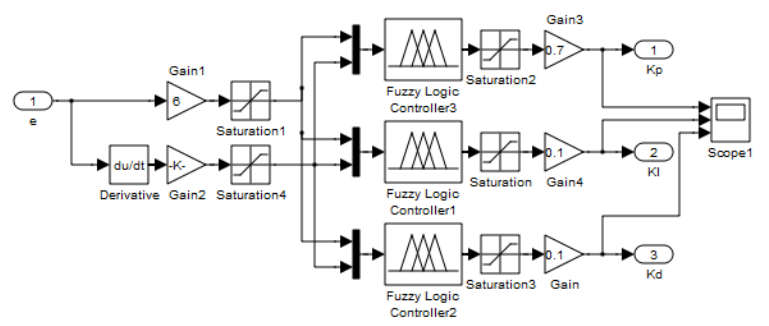

(c) the structure of fuzzy PID

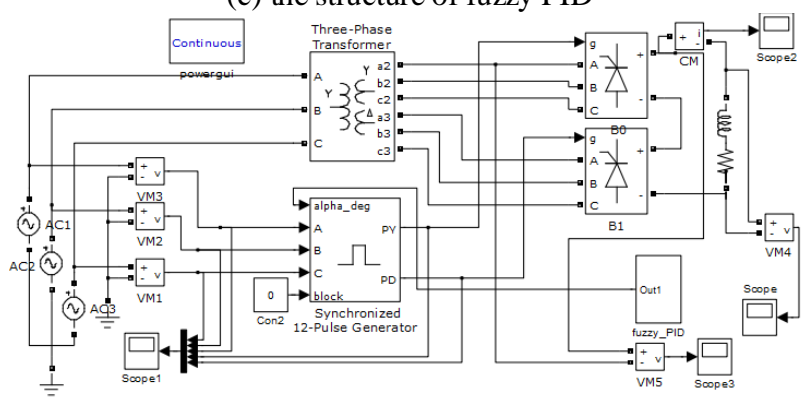

(d) the simulation model

Figure 4. the simulation of 12-pulse rectifier control system

It's modeling and simulating[10] 12 pulse rectifier circuit in Mat lab, the simulation model as Fig .4(d).

The main circuit has three-phase AC voltage source, rectifier transformers, series connection thyristor rectifier bridge, trigger pulse and RL load components. The setting of simulation model are as follows: 
$\mathrm{T} 1$ and $\mathrm{T} 2$ are series connection structure, $\mathrm{T}$ is the rectifier transformer. Secondary side uses Y-shaped and $\triangle$ -shaped connection, the phase voltage difference is $30^{\circ}$. Three-phase AC voltage source amplitude is $380 \mathrm{~V}$, phase respectively $120^{\circ}, 0^{\circ},-120^{\circ}, \mathrm{RL}$ load is set as $\mathrm{R}=$ $10 \mathrm{ohm}, \mathrm{L}=0.15 \mathrm{H}$, the trigger pulse frequency is $50 \mathrm{~Hz}[11]$, getting the trigger angle by fuzzy PID control.

\section{A. Simulation results}

The simulation results is shown as Fig. 5(a) (d). The step response of fuzzy PID control shows as Fig. 5 (a). When the angle of the phase-shift trigger pulse is $0^{\circ}$, voltage waveform of 12 pulse rectifier resistive inductive load shown as Fig. 5(b). When the angle of the phase-shift trigger pulse is $30^{\circ}$, the voltage waveform 12 pulse rectifier load on the sense resistor shown as Fig. 5(c). When the angle of the phase-shift trigger pulse is $30^{\circ}$, the trigger pulse thyristor rectifier VT on Fig. 5(d).

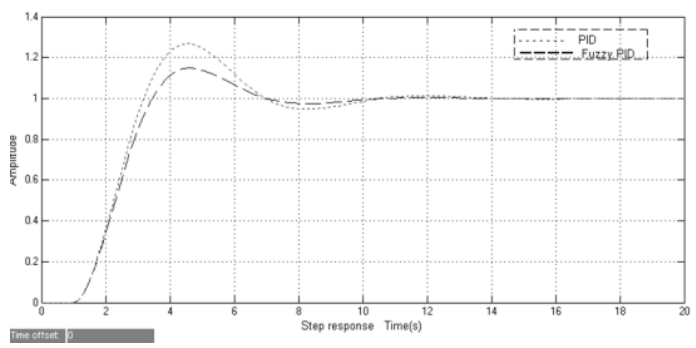

(a) Step response

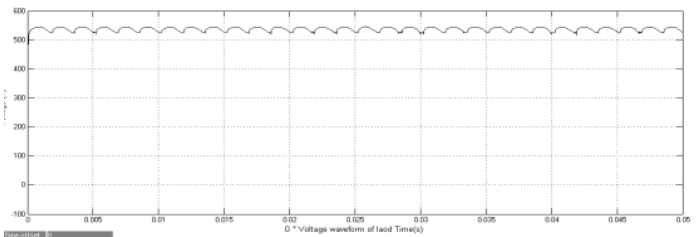

(b)voltage waveforms in resistive inductive load at $0^{\circ}$

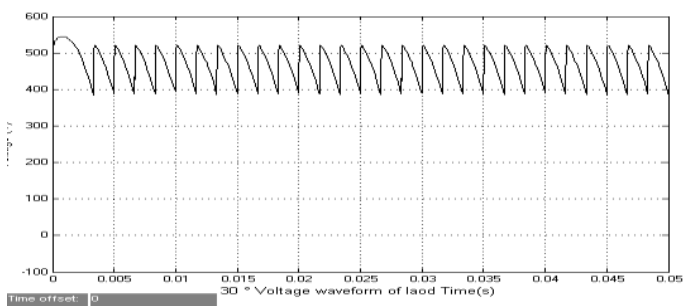

(c) Voltage waveforms in resistive inductive load at $30^{\circ}$

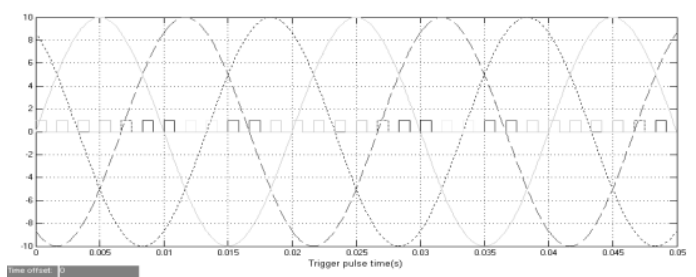

(d) Trigger pulse at $30^{\circ}$

Figure 5. simulation results

\section{B. Analysis of simulation results}

From the Figure above step response with fuzzy PID control, it can be seen, fuzzy PID response speed has increased, overshoot and settling time reduced significantly compared with conventional PID. Simulation results show that: the fuzzy PID control strategy for the 12pulse rectifier control, can be achieve the desired control relatively Rectifier.

\section{CONCLUSIONS}

In view of our study above, we can come to the conclusion below:

1) It is proposed a advanced control theory of fuzzy PID control strategy. According to experts control rules and decision tables, determine the size of the control quantity, fuzzy control and flexibility, and robustness and high accuracy of traditional PID control.

2) By simulating 12 pulse rectification control circuitry based on fuzzy PID control strategy, results show that compared to conventional PID, the fuzzy PID control system has smaller overshoot, faster dynamic response and greater stability.

3) It can realize efficient control of controller objects of large power rectifier electronics equipment which have varied application and changing load, and achieve the fuzzy PID control for nonlinear systems in theory. The design not only enhance the quality of rectifier equipment, but also have important significance to achieve the rectifier device control.

\section{REFERENCE}

[1] Yong Li, Longfu Luo, Rehtanz, C, et al. An industrial dc power supply systembased on an inductive filtering method.[J] IEEE Transactions on IndustrialElectronics, 2012, 59(2):714-719.

[2] Liu Hongda, Yue Wen-jie, Shen Nai-jun. Fault Detection Method in Micro-Grid Multi-Pulse Thyristor Rectifier Circuit. [J] IEEE, 2012, 23(9) : 1-5 [3].

[3] Zhang Dewang, S.Predictive Fast DSP-Based Current Controller for 12-Pulse Hybrid-Mode Thyristor Rectifier.[J] IEEE, 2013 75(45) : 145-149.

[4] Wang ZhaoAn, Huang Jun Power electronics technology (The Fourth Edition) .[M] Beijing: Mechanical Industry Press.78-79.

[5] Ning Zhihao, Luo Longfu. The comprehensive energy research theory and new technology of power rectifier systems $\mathrm{PhD}$ thesis: Hunan University 11-13. (2012).

[6] Zhihao Ning, Longfu Luo, Jiazhu Xu, et al. Technical analysis and synthesis energysaving design of the high power DC power supply system. 2010 InternationalConference on Power System Technology, 24-27 Oct. 2010, Hangzhou, China, pp.1-6.

[7] Qin Mao,Xu Xiaofang, Sun Xinzhi .FPGA based implementation of phase-shifted trigger controller for SCR.[J]. Electrical Measurement \& Instruementation, 2014 51(3): 77-80.

[8] Yang Xue, Peng Guangzheng, Fan Meng, Wu Qinghe. New Asymmetric Fuzzy PID Control for Pneumatic Position Control System[J]. Journal of Beijing Institute of Technology, 2010, 13(1): 29-33.

[9] CHANGA W D, HWANGB R C, HSIEHA J G.A self-tuning PID control for a class of nonlinear systems based on the Lyapunov approach[J]. Journal of proeess Control, 2010, 12(2): 233-242.

[10] Zhang meng,Zhang Qiang,Wang hong The research of fuzzy neural network applied in induction heating power supply.[J] Industry Control and Application, 2014 33(7) :13-16.

[11] Wang Enliang The temperature control system design of medium frequency induction furnace based on the fuzzy PID. $[\mathrm{J}]$ Micromachine and Application ,2014 33(24) 74-76. 